\title{
PHENOLIC COMPOUNDS FROM CALLISTEMON CITRINUS LEAVES AND STEMS
}

\author{
Pham Ngoc Khanh ${ }^{1}$, Ho Viet Duc ${ }^{2}$, Tran Thu Huong ${ }^{1}$, Vu Thi Ha ${ }^{1}$, Doan Thi Van ${ }^{1}$, \\ Ninh The Son ${ }^{1}$, Young Ho Kim ${ }^{3}$, Do Quoc Viet ${ }^{4}$, Nguyen Manh Cuong, ${ }^{1}$ * \\ ${ }^{I}$ Department of Bioactive Compounds, Institute of Natural Products Chemistry, Vietnam \\ Academy of Science and Technology, 18 Hoang Quoc Viet, Cau Giay, Hanoi \\ ${ }^{2}$ Faculty of Pharmacy, Hue University of Medicine and Pharmacy, Hue University, \\ 06 Ngo Quyen, Hue, Vietnam \\ ${ }^{3}$ College of Pharmacy, Chungnam National University, Daejeon 305-764, Korea \\ ${ }^{4}$ Institute of Chemistry, Vietnam Academy of Science and Technology, 18 Hoang Quoc Viet, \\ Cau Giay, Hanoi \\ "Email:nnmcuong_inpc@yahoo.com.vn
}

Received: 25 June 2015; Accepted for publication: 2 January 2016

\begin{abstract}
In the search for bioactive constituents from Vietnamese plants, the leaves and stems of Callistemon citrinus (Curtis) Skeels were selected for chemical investigation. Phytochemical analysis of plant led to the isolation of eight phenolic compounds including two flavonoids (eucalyptine (1) and 8-demethyleucalyptine (2)), two alcohols (blumenol A (3), ntetratriacontanol (4)), three benzoic acid derivatives (acid gallic (5), methyl gallate (6) protocatechuic acid (7)), one sterol $(\beta$-sitosterol $(\mathbf{8}))$, and along with one sesquiterpene $(2,6,10$ bisabolatriene (9)). The structures of the natural compounds were determined from the spectroscopic evidences including 1D- and 2D-NMR and ESI-MS.

Keywords: Callistemon citrinus (Curtis) Skeels, flavonoid, triterpenoid.

\section{INTRODUCTION}

The Myrtaceae family in Vietnam comprises about 30 genera, several of which are very common and widely distributed throughout the country such as Eucalyptus, Rhodomyrtus, Syzygium, and Callistemon [1]. Species Callistemon citrinus (Curtis) Skeels, local name "Tram bong do", is grown as ornaments in Vietnam for its beautiful form, glossy green foliage and red, bottle-brush like flowers. Phytochemical studies of Callistemon species have led to the isolation
\end{abstract}


and characterization of flavones [2], acylphloroglucinols [3, 4], triterpenoids [5], neolignans [6], essential oil, steroids and saponins [7].

In previous papers, we reported the isolation of acylphloroglucinol derivatives and triterpenoids with soluble epoxide hydrolase inhibitory activity [8] as well as flavonoids with inhibitory effect on NO production in LPS-activated RAW264.7 macrophage [9] from Callistemon citrinus leaves and stems. In this study, we describe the isolation and structural elucidation of eight phenolic compounds, including two flavones (eucalyptin (1) and 8demethyleucalyptin (2)), two alcohols (blumenol A (3), tetratriacontan-1-ol (4)), three benzoic acid derivatives (gallic acid (5), methyl gallate (6) and protocatechuic acid (7)), one sterol $(\beta$ stigmasterol (8)) along with one sesquiterpene (2,6,10-bisabolatriene (9)). The structures of the natural compounds were identified by spectroscopic evidences including 1D- and 2D-NMR and ESI-MS.

\section{MATERIALS AND METHODS}

\subsection{General experimental procedures}

${ }^{1} \mathrm{H}-\mathrm{NMR}(500 \mathrm{MHz}),{ }^{13} \mathrm{C}$ NMR $(125 \mathrm{MHz})$ spectra were measured on a Bruker AVANCE 500 spectrometer. The ESI-MS spectra were obtained with a ESI-MicroQ-TOF III (Bruker Daltonics Inc.) and a FT-ESI-MS (Varian Inc.) mass spectrometer. UV and IR spectra were obtained on a JASCO V-630 and an Impact 410 Nicolet FT-IR spectrometer, respectively. Column chromatography (CC) was carried out on silica gel ( $\mathrm{Si} 60 \mathrm{~F}_{254}, 230-400$ mesh, Merck). All solvents were distilled before use. Precoated plates of silica gel $60 \mathrm{~F}_{254}$ were used for analytical purposes. Compounds were visualized under UV radiation $(254,365 \mathrm{~nm})$ and by spraying plates with $10 \% \mathrm{H}_{2} \mathrm{SO}_{4}$ followed by heating with a heat gun.

\subsection{Plant material}

The leaves and stems of Callistemon citrinus (Curtis) Skeels were collected in Hue province, Vietnam. The plants were identified by the botanist Dr. Tran The Bach (Institute of Ecology and Biological Resources, VAST). A voucher specimen (HCTN-2118) is deposited in the herbarium of the Institute of Natural Products Chemistry, VAST, Hanoi, Vietnam.

\subsection{Extraction and isolation}

Dried powdered leaves and stems of $C$. citrinus $(3.2 \mathrm{~kg}$ ) were extracted with $\mathrm{MeOH}$ over the period of 5 days at room temperature and concentrated under reduced pressure to yield a black crude $\mathrm{MeOH}$ extract $(190 \mathrm{~g})$. This crude $\mathrm{MeOH}$ extract was suspended in hot $\mathrm{MeOH}-$ water $(1: 1, \mathrm{v} / \mathrm{v})$ and successively partitioned with $n$-hexane, dichloromethane (DCM), ethyl acetate (EtOAc) and water. The resulting fractions were concentrated under reduced pressure to give the corresponding solvent-soluble fractions $n$-hexane (27.3 g), DCM (63.0 g), EtOAc (55.4 $\mathrm{g})$, and water.

The $n$-hexane fraction $(27.0 \mathrm{~g})$ was chromatographed on a silica gel column, using solvent gradients of $n$-hexane - EtOAc $(1: 0,40: 1,20: 1,10: 1$, and 5:1, v/v, $1.0 \mathrm{~L}$ each) to afford 5 subfractions (H1 to H5). The sub-fraction $\mathrm{H} 4$ was rechromatographed on silica gel column, eluting with DCM - EtOAc (20:1, v/v) to yield compound $1(11.0 \mathrm{mg})$ and sub-fraction H4A, which was further purified on a silica gel column eluting with $\mathrm{CHCl}_{3}-\mathrm{EtOAc}(15 / 1, \mathrm{v} / \mathrm{v})$ to get 
compound $9(5.6 \mathrm{mg})$. A precipitate from the sub-fraction $\mathrm{H} 3$ was filtered and washed by $n$ hexane $(1 \mathrm{~mL} \times 2)$ to yield $4(12.8 \mathrm{mg})$. The residue was separated by $\mathrm{CC}$ on silica gel, eluting with acetone- $\mathrm{MeOH}(1 / 4, \mathrm{v} / \mathrm{v})$ to give compound $7(15.7 \mathrm{mg})$ and $\mathbf{8}(7.2 \mathrm{mg})$.

The DCM fraction $(63.0 \mathrm{~g})$ was subjected to chromatography on a flash silica gel column (400 - 630 mesh), eluted with gradient DCM - methanol (1:0, 40:1, 20:1, 10:1, 5:1, 2.5:1, 1:1 and $0: 1, \mathrm{v} / \mathrm{v}, 1.5 \mathrm{~L}$ each) to afford 6 subfractions (Fr. D1 to D6). The subfraction D1 (10.2 g) was subjected to silica gel CC, eluted with an isocratic solvent mixture of $n$-hexane-DCMacetone $(1: 2: 0.1, \mathrm{v} / \mathrm{v} / \mathrm{v})$, to afford 12 subfractions (D1A to D1L). The subfraction D1C was eluted with isocratic solvent system of $n$-hexane - DCM (1:3, v/v) on a silica gel column (230 $400 \mathrm{mesh}$ ) to yield compound 2 (6.1 $\mathrm{mg})$.

The EtOAc fraction (55.4 g) was chromatographed on a flash silica gel column (400 - 630 mesh) eluting with gradients of $\mathrm{CH}_{2} \mathrm{Cl}_{2}-\mathrm{MeOH}(1: 0 \sim 0: 1, \mathrm{v} / \mathrm{v})$ to afford 7 subfractions (E1 E7). The subfraction E3 was subjected to column chromatography on silica gel eluting with $\mathrm{CH}_{2} \mathrm{Cl}_{2}$ $\mathrm{MeOH}(15: 1)$ to afford 2 subfractions E3A and E3B. The subfraction E3B was further separated on a silica gel column eluting with a mixture of $n$-hexane $-\mathrm{Me}_{2} \mathrm{CO}(2: 1)$, to yield compounds 6 $(99.0 \mathrm{mg})$ and $\mathbf{3}(4.9 \mathrm{mg})$. The subfraction $\mathrm{E} 4$ was subjected to column chromatography on silica gel eluting with gradients of $\mathrm{CHCl}_{3}-\mathrm{MeOH}-\mathrm{H}_{2} \mathrm{O}(4: 1: 0.1-3: 1: 0.1, \mathrm{v} / \mathrm{v} / \mathrm{v})$ to obtain 6 subfractions (E4A - E4F). The subfraction $\mathrm{E} 4 \mathrm{C}$ was subjected to $\mathrm{CC}$ on silica gel eluting with an isocratic mixture of $\mathrm{Me}_{2} \mathrm{CO}-\mathrm{CHCl}_{3}-\mathrm{H}_{2} \mathrm{O}(2: 1: 0.1, \mathrm{v} / \mathrm{v} / \mathrm{v})$, to afford 6 subfractions $(\mathrm{E} 4 \mathrm{C} 1 \sim \mathrm{E} 4 \mathrm{C} 6)$. The subfraction E4C1 was chromatographed over a RP-18 column, eluting with $\mathrm{MeOH}-\mathrm{H}_{2} \mathrm{O}$ $(1: 1, \mathrm{v} / \mathrm{v})$ to obtain 4 subfractions (C1A C1D). The subfraction C1A was rechromatographed over a RP-18 column eluting with $\mathrm{MeOH}-\mathrm{H}_{2} \mathrm{O}$ (1:3) to yield compounds 5 (123.0 mg).

\subsection{Spectral and physical data}

2.4.1 Eucalyptin (1) pale lemon yellow powder, $\mathrm{C}_{19} \mathrm{H}_{18} \mathrm{O}_{5}(\mathrm{M}=326) .{ }^{1} \mathrm{H}-\mathrm{NMR}(500 \mathrm{MHz}$, $\left.\mathrm{CDCl}_{3}\right), \delta(\mathrm{ppm}): 6.60$ (s, 1H, H-3), 7.85 (d, 2H, $\left.J=9.5 \mathrm{~Hz}, \mathrm{H}-2^{\prime}, 6^{\prime}\right), 7.01$ (d, 2H, $J=9.5 \mathrm{~Hz}, \mathrm{H}-$ $\left.3^{\prime}, 5^{\prime}\right), 12.87(\mathrm{~s}, 5-\mathrm{OH}), 3.80\left(\mathrm{~s}, 3 \mathrm{H}, 7-\mathrm{OCH}_{3}\right), 3.89$ (s, 3H, 4'- $\left.\mathrm{OCH}_{3}\right), 2.20\left(\mathrm{~s}, 3 \mathrm{H}, 8-\mathrm{CH}_{3}\right), 2.38$ $\left(\mathrm{s}, 3 \mathrm{H}, 6-\mathrm{CH}_{3}\right) .{ }^{13} \mathrm{C}-\mathrm{NMR}\left(125 \mathrm{MHz}, \mathrm{CDCl}_{3}\right), \delta(\mathrm{ppm}): 163.8$ (s, C-2), $104.0(\mathrm{~d}, \mathrm{C}-3), 183.2$ (s, C-4), 157.3 (s, C-5), 114.1 (s, C-6), 162.6 (s, C-7), 107.4 (s, C-8), 152.9 (s, C-9), 108.8 (s, C10), 123.8 (s, C-11), 127.9 (d, C-2',6'), 114.5 (d, C-3',5'), 159.8 (s, C-4'), 60.4 (q, 7- $\mathrm{OCH}_{3}$ ), $55.5\left(\mathrm{q}, 4^{\prime}-\mathrm{OCH}_{3}\right), 8.5\left(\mathrm{q}, 8-\mathrm{CH}_{3}\right), 8.3\left(\mathrm{q}, 6-\mathrm{CH}_{3}\right)$. ESI-MS $(\mathrm{m} / \mathrm{z}): 327[\mathrm{M}+\mathrm{H}]^{+}$.

2.4.2 8-demethyleucalyptin (2) white powder, $\mathrm{C}_{18} \mathrm{H}_{16} \mathrm{O}_{5}(\mathrm{M}=312)$. ${ }^{1} \mathrm{H}-\mathrm{NMR}$ (500 MHz, DMSO$\left.d_{6}\right), \delta(\mathrm{ppm}): 6.86(\mathrm{~s}, 1 \mathrm{H}, \mathrm{H}-3), 6.92(\mathrm{~s}, 1 \mathrm{H}, \mathrm{H}-8), 8.05$ (d, 2H, $\left.J=9.5 \mathrm{~Hz}, \mathrm{H}-2^{\prime}, 6^{\prime}\right), 7.10$ (d, 2H, $\left.J=9.0 \mathrm{~Hz}, \mathrm{H}-3^{\prime}, 5^{\prime}\right), 13.05$ (s, 5-OH), $3.86\left(\mathrm{~s}, 3 \mathrm{H}, 7-\mathrm{OCH}_{3}\right), 3.91$ (s, 3H, 4'-OCH 3$), 1.99(\mathrm{~s}, 3 \mathrm{H}$, 6- $\left.\mathrm{CH}_{3}\right) .{ }^{13} \mathrm{C}-\mathrm{NMR}\left(125 \mathrm{MHz}, \mathrm{DMSO}-d_{6}\right), \delta(\mathrm{ppm}): 163.0$ (s, C-2), 103.7 (d, C-3), 181.9 (s, C-4), 162.3 (s, C-5), 107.5 (s, C-6), 163.3 (s, C-7), 90.3(s, C-8), 155.4 (s, C-9), 104.4 (s, C-10), 122.8 (s, C-1'), 128.2 (d, C-2', 6'), 114.5 (d, C-3', 5'), 157.4 (s, C-4'), 56.3 (q, 7-OCH 3 ), 55.5 (q, 4'$\left.\mathrm{OCH}_{3}\right), 7.2\left(\mathrm{q}, 6-\mathrm{CH}_{3}\right)$. ESI-MS $(\mathrm{m} / \mathrm{z}): 313[\mathrm{M}+\mathrm{H}]^{+}$.

2.4.3 Blumenol $A$ (3) white powder, $\mathrm{C}_{13} \mathrm{H}_{20} \mathrm{O}_{3}(\mathrm{M}=224)$. ${ }^{1} \mathrm{H}-\mathrm{NMR}$ (500 MHz, MeOD$\left.d_{4}\right), \delta(\mathrm{ppm}): 2.50(1 \mathrm{H}, \mathrm{d}, J=17.0 \mathrm{~Hz}, \mathrm{H}-6 \mathrm{a}), 2.19(1 \mathrm{H}, \mathrm{dd}, J=6.5,17.0 \mathrm{~Hz}, \mathrm{H}-6 \mathrm{~b}), 5.90(1 \mathrm{H}$, $\mathrm{m}, J=1.5 \mathrm{~Hz}, \mathrm{H}-2), 5.80(1 \mathrm{H}, \mathrm{m}, J=16.0 \mathrm{~Hz}, \mathrm{H}-7), 5.82(1 \mathrm{H}, \mathrm{m}, J=16.0,4.5 \mathrm{~Hz}, \mathrm{H}-8), 4.34$ $(1 \mathrm{H}, \mathrm{m}, J=4.5 ; 6.5 \mathrm{~Hz}, \mathrm{H}-9), 1.26(3 \mathrm{H}, \mathrm{d}, J=6.5 \mathrm{~Hz}, \mathrm{H}-10), 1.04(3 \mathrm{H}, \mathrm{s}, \mathrm{H}-11), 1.06(3 \mathrm{H}, \mathrm{s}, \mathrm{H}-$ 12), $1.93(3 \mathrm{H}, \mathrm{d}, J=1.5 \mathrm{~Hz}, \mathrm{H}-13) .{ }^{13} \mathrm{C}-\mathrm{NMR}$ (125 MHz, MeOD- $\left.d_{4}\right), \delta$ (ppm): $201.2(\mathrm{~s}, \mathrm{C}-1)$, 
42.4 (s, C-5), 50.7 (t, C-6), 127.1 (s, C-2), 167.4 (s, C-3), 79.9 (s, C-4), 129.9 (d, C-7), 136.9 (d, C-8), 68.6 (d, C-9), 23.8 (q, C-10), 24.5 (q, C-11), 23.4 (q, C-12), 19.5 (q, C-13).

2.4.4 Tetratriacontan-1-ol (4) white powder, $\mathrm{C}_{34} \mathrm{H}_{70} \mathrm{O}(\mathrm{M}=494)$. ${ }^{1} \mathrm{H}-\mathrm{NMR}\left(500 \mathrm{MHz}, \mathrm{CDCl}_{3}\right)$ : $3.64(2 \mathrm{H}, \mathrm{t}, J=6.5 \mathrm{~Hz}, \mathrm{H}-1), 1.55(4 \mathrm{H}, \mathrm{m}, \mathrm{H}-2 \& 3), 1.25-1.34(60 \mathrm{H}, \mathrm{H}-4 \div \mathrm{H}-33), 0.88$ (3H, t, $J=$ $6.5 \mathrm{~Hz}, \mathrm{H}-34)$; ESI-MS (m/z): $493[\mathrm{M}-\mathrm{H}]^{-}$.

2.4.5. Gallic acid (5) white powder, $\mathrm{C}_{7} \mathrm{H}_{6} \mathrm{O}_{5}(\mathrm{M}=170)$. ${ }^{1} \mathrm{H}-\mathrm{NMR}\left(500 \mathrm{MHz}, \mathrm{MeOD}-d_{4}\right), \delta$ (ppm): $7.00(2 \mathrm{H}, \mathrm{s}, \mathrm{H}-2,-6) .{ }^{13} \mathrm{C}-\mathrm{NMR}\left(125 \mathrm{MHz}, \mathrm{MeOD}-d_{4}\right), \delta(\mathrm{ppm}): 170.5$ (s, C-7), $110.4(\mathrm{~d}, \mathrm{C}-2,-$ 6), 146.5 (s, C-3, -5), 139.7 (s, C-4), 122.0 (s, C-1).

2.4.6. Methyl gallate (6) white powder, $\mathrm{C}_{8} \mathrm{H}_{8} \mathrm{O}_{5}(\mathrm{M}=184) .{ }^{1} \mathrm{H}-\mathrm{NMR}\left(500 \mathrm{MHz}, \mathrm{MeOD}-d_{4}\right)$, $\delta$ (ppm): $7.00(2 \mathrm{H}, \mathrm{s}, \mathrm{H}-2,-6), 3.77(3 \mathrm{H}, \mathrm{s}, \mathrm{H}-8) .{ }^{13} \mathrm{C}-\mathrm{NMR}$ : $\left(500 \mathrm{MHz}, \mathrm{MeOD}-d_{4}\right), \delta(\mathrm{ppm})$ : 169.1 (s, C-7), 52.3 (q, C-8), 110.1 (d, C-2, -6), 146.6 (s, C-3, -5), 139.8 (s, C-4), 121.5 (s, C-1).

2.4.7. Protocatechuic acid (7) white powder, $\mathrm{C}_{7} \mathrm{H}_{6} \mathrm{O}_{4},(\mathrm{M}=154)$. ${ }^{1} \mathrm{H}-\mathrm{NMR}(500 \mathrm{MHz}, \mathrm{MeOD}-$ $\left.d_{4}\right), \delta(\mathrm{ppm}): 7.93(\mathrm{COOH}), 7.45(1 \mathrm{H}, \mathrm{s}, \mathrm{H}-2), 6.81(1 \mathrm{H}, \mathrm{d}, J=8.0 \mathrm{~Hz}, \mathrm{H}-5), 7.44(1 \mathrm{H}, \mathrm{d}, J=8.0$ $\mathrm{Hz}, \mathrm{H}-6) .{ }^{13} \mathrm{C}-\mathrm{NMR}\left(125 \mathrm{MHz}, \mathrm{MeOD}-d_{4}\right), \delta$ (ppm): 170.4 (s, C-7), 115.8 (d, C-6), 117.8 (d, C5), 123.4 (s, C-4), 146.1 (s, C-3), 123.9 (d, C-2), 151.5 (s, C-1).

2.4.8. $\beta$-sitosterol $(8)$ white powder, $\mathrm{C}_{29} \mathrm{H}_{50} \mathrm{O}(\mathrm{M}=414) .{ }^{1} \mathrm{H}-\mathrm{NMR}\left(500 \mathrm{MHz}, \mathrm{CDCl}_{3}\right), \delta$ (ppm): $3.52(1 \mathrm{H}, \mathrm{tt}, J=6.5,11.0 \mathrm{~Hz}, \mathrm{H}-3), 5.35(1 \mathrm{H}, \mathrm{brd}, J=6.5 \mathrm{~Hz}, \mathrm{H}-6), 0.68(3 \mathrm{H}, \mathrm{s}, \mathrm{H}-18), 1.01$ $(3 \mathrm{H}, \mathrm{s}, \mathrm{H}-19), 0.90(3 \mathrm{H}, \mathrm{d}, J=7.0 \mathrm{~Hz}, \mathrm{H}-21), 0.83(3 \mathrm{H}, \mathrm{d}, J=6.5 \mathrm{~Hz}, \mathrm{H}-26), 0.81(3 \mathrm{H}, \mathrm{d}, J=$ $7.0 \mathrm{~Hz}, \mathrm{H}-28), 0.85$ (3H, d, $J=7.0 \mathrm{~Hz}, \mathrm{H}-29)$.

2.4.9. 2,6,10-bisabolatriene (9) colourless oil, $\mathrm{C}_{15} \mathrm{H}_{24}(\mathrm{M}=204) .{ }^{1} \mathrm{H}-\mathrm{NMR}\left(500 \mathrm{MHz}, \mathrm{CDCl}_{3}\right)$, $\delta$ (ppm): $2.01(2 \mathrm{H}$, quint. $J=7.5 \mathrm{~Hz}, \mathrm{H}-1) ; 5.10(1 \mathrm{H}, \mathrm{H}-2) ; 2.07(2 \mathrm{H}$, quint., $J=7.5 \mathrm{~Hz}, \mathrm{H}-4)$; 2.07 (2H, quint., $J=7.5 \mathrm{~Hz}, \mathrm{H}-5$ ); 1.99 (2H, quint., $J=8.0 \mathrm{~Hz}, \mathrm{H}-8) ; 1.99$ (2H, quint., $J=8.0$ Hz, H-9); 5.10 (1H, H-10); 1.60 (3H, s, H-12); 1.60 (3H, s, H-13); 1.60 (3H, s, H-14); 1.68 (3H, $\mathrm{s}, \mathrm{H}-15) .{ }^{13} \mathrm{C}-\mathrm{NMR}\left(125 \mathrm{MHz}, \mathrm{CDCl}_{3}\right), \delta(\mathrm{ppm}): 26.7$ (t, C-1); 124.3 (d, C-2); 135.1 (s, C-3); 28.3 (t, C-4); 26.8 (t, C-5); 124.2 (s, C-6); 134.9 (s, C-7); 39.8 (t, C-8); 39.7 (t, C-9); 124.4 (d, C-10); 131.2 (s, C-11); 16.1 (q, C-12); 16.0 (q, C-13); 25.7 (q, C-14); 17.7 (q, C-15) ; ESI-MS $(\mathrm{m} / \mathrm{z}): \mathrm{m} / z 205[\mathrm{M}+\mathrm{H}]^{+}$.

\section{RESULTS AND DISCUSSION}

Compound (2) was isolated from the dichloromethane (DCM) fraction of the methanolic extract of Callistemon citrinus leaves and stems. Three compounds $(\mathbf{3}, \mathbf{5}$ and $\mathbf{6})$ were isolated from the EtOAc fraction and five compounds $(1,4,7,8$ and 9$)$ were obtained from the $n$-hexane fraction.

Compounds 1 and $\mathbf{2}$ are two flavones (Fig. 1). While compound $\mathbf{1}$ was obtained from $n$ hexane fraction, compound $\mathbf{2}$ was isolated from EtOAc fraction of the methanolic extract of $C$. citrinus leaves. The 1D-NMR spectra including ${ }^{1} \mathrm{H},{ }^{13} \mathrm{C}$ and DEPT of two compounds are very similar. The ${ }^{1} \mathrm{H}$ spectrum of 1 showed a low-field singlet signal $\left(\delta_{\mathrm{H}} 12.87,5-\mathrm{OH}\right)$, two doublet aromatic proton signals characteristic of a para-substituted B-ring, two methoxy groups $\left(\delta_{\mathrm{H}}\right.$ 3.89, and 3.80) and two singlet methyl substituents $\left(\delta_{\mathrm{H}} 2.38\right.$ and 2.20$) .{ }^{13} \mathrm{C}-\mathrm{NMR}$ analysis of 1 revealed the presence of 19 carbons including one carbonyl $\left(\delta_{\mathrm{C}} 183.2\right)$, four methines of a para- 
substituted B-ring, one A-ring aromatic methine, two methoxyl, and two C-methyl carbons. Molecular formula of $\mathbf{1}$ was determined to be $\mathrm{C}_{19} \mathrm{H}_{18} \mathrm{O}_{5}$ based on the quasi - molecular ion peak observed at $\mathrm{m} / z 327[\mathrm{M}+\mathrm{H}]^{+}$in positive mode electrospray ionization (ESI-MS) mass spectrometry. From the 1D-NMR and ESI-MS spectral data, compound 1 was identified as 5hydroxy-7,4'-dimethoxy-6,8-dimethylflavone with common name as eucalyptin [10]. Similarly, molecular formula of 2 was $\mathrm{C}_{18} \mathrm{H}_{16} \mathrm{O}_{5}$, based on the ESI-MS quasi - molecular ion peak at $\mathrm{m} / \mathrm{z}$ $313[\mathrm{M}+\mathrm{H}]^{+}$, indicating the absence of one methyl group in comparison with that of eucalyptin (1). In the NMR spectra of 2, the absence of one C-methyl signal, resulting in the shift of C-8 signal to the upfield region $\left(\delta_{\mathrm{C}} 90.3\right.$ in 2 vs. 107.4 in $\left.\mathbf{1}\right)$, and the addition of one proton in the aromatic field $\left(\delta_{\mathrm{H}} 6.92, \mathrm{H}-8\right)$, as well as by comparison with spectral data reported in the literature, confirmed the structure of compound 2 as 5-hydroxy-7,4'-dimethoxy-6-methylflavone or 8-demethyleucalyptin [10].

Compound 3 was isolated as white powder from the EtOAc fraction. The ${ }^{1} \mathrm{H}-\mathrm{NMR}$ spectrum of 3 showed characteristic signals of four methyl groups at $\delta_{\mathrm{H}} 1.26(3 \mathrm{H}, \mathrm{d}, J=6.5 \mathrm{~Hz}$, $\mathrm{H}-10), 1.04(3 \mathrm{H}, \mathrm{s}, \mathrm{H}-11), 1.06(3 \mathrm{H}, \mathrm{s}, \mathrm{H}-12)$, and $1.93(3 \mathrm{H}, \mathrm{d}, J=1.5 \mathrm{~Hz}, \mathrm{H}-13)$; one methylene at $\delta_{\mathrm{H}} 2.50(1 \mathrm{H}, \mathrm{d}, J=17.0 \mathrm{~Hz}, \mathrm{H}-6 \mathrm{a}), 2.19(1 \mathrm{H}, \mathrm{d}, J=17.0 ; 6.5 \mathrm{~Hz}, \mathrm{H}-6 \mathrm{~b})$ and four methines at $\delta_{\mathrm{H}} 5.90(1 \mathrm{H}, \mathrm{m}, \mathrm{H}-2), 5.80(1 \mathrm{H}, \mathrm{d}, J=16.0 \mathrm{~Hz}, \mathrm{H}-7), 5.82(1 \mathrm{H}, \mathrm{dd}, J=16.0,4.5 \mathrm{~Hz}, \mathrm{H}-8), 4.34$ $(1 \mathrm{H}, \mathrm{m}, J=6.5,4.5 \mathrm{~Hz}, \mathrm{H}-9)$. The strong coupling constant $(J=16.0 \mathrm{~Hz})$ of protons $\mathrm{H}-7$ and $\mathrm{H}-$ 8 indicated the trans-configuration of $\mathrm{C}_{7}=\mathrm{C}_{8}$ double bond. ${ }^{13} \mathrm{C}$-NMR/DEPT spectra of $\mathbf{3}$ showed 13 cacbons $\left(1 \times \mathrm{CO}, 3 \times \mathrm{C}_{4}, 4 \times \mathrm{CH}, 4 \times \mathrm{CH}_{3}\right.$ and $\left.1 \times \mathrm{CH}_{2}\right)$. The analysis of $\mathrm{HMBC}$ spectrum indicated the presence of a 3,5,5-trimethyl-2-cyclohexene-1-one unit and a 3-hydroxy-1-butenyl side chain. Comparison of the spectral data with those in the literature, compound $\mathbf{3}$ was determined as blumenol A [11].<smiles>[R]c1c(OC)c(C)c(O)c2c(=O)cc(-c3ccc(OC)cc3)oc12</smiles>

$\mathrm{R}=\mathrm{CH}_{3} \quad$ Eucalyptin (1)

$\mathrm{R}=\mathrm{H} \quad$ 8-demethyleucalyptin (2)

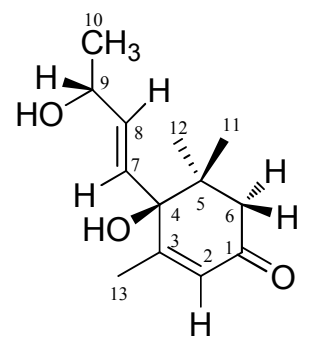

Blumenol A (3)<smiles>[R]c1cc(C(=O)OC)cc(O)c1O</smiles>

$\mathrm{R}=\mathrm{OH}, \mathrm{R}_{1}=\mathrm{H}$ gallic acid (5)

$\mathrm{R}=\mathrm{OH}, \mathrm{R}_{1}=\mathrm{Me}$ methyl gallate (6)

$\mathrm{R}=\mathrm{H}, \quad \mathrm{R}_{1}=\mathrm{H} \quad$ protocatechuic acid $(\mathbf{7})$

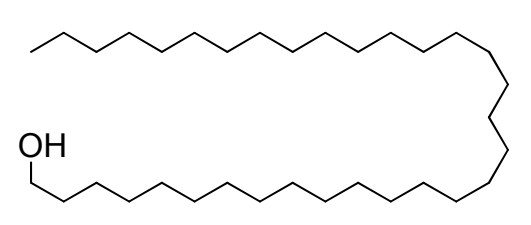

Tetratriacontan-1-ol (4)<smiles></smiles><smiles>CC(C)=CCCC(C)=C1CC=C(C)CC1</smiles>

2,6,10-bisabolatriene (9)

Figure 1. Structures of isolated compounds (1-9).

Compound 4 was obtained as white powder from $n$-hexane fraction. ${ }^{1} \mathrm{H}$-NMR spectrum of 4 presented signals of hydroxymethylene protons at $\delta_{\mathrm{H}} 3.64(2 \mathrm{H}, \mathrm{t}, J=7.0 \mathrm{~Hz}, \mathrm{H}-1)$; two methylenes at $\delta_{\mathrm{H}} 1.56\left(4 \mathrm{H}, \mathrm{t}, J=7.0 \mathrm{~Hz}, \mathrm{H}_{2}-2,3\right)$, sixty consecutive methylene protons at $\delta_{\mathrm{H}} 1.25$ $-1.26(60 \mathrm{H}, \mathrm{s})$ and one methyl group $\left(\delta_{\mathrm{H}} 0.88,3 \mathrm{H}, \mathrm{t}, J=7.0 \mathrm{~Hz}, \mathrm{H}-34\right)$. These data suggested 
that 4 was a long-chain alcohol. The ESI-MS spectrum of $\mathbf{4}$ in negative mode showed a pseudomolecular ion peak at $m / z 493[\mathrm{M}-\mathrm{H}]^{-}$, indicating the molecular formula of $\mathbf{4}$ as $\mathrm{C}_{34} \mathrm{H}_{70} \mathrm{O}$. Compound 4 was determined to be $n$-tetratriacontanol, other name sapiol [12].

Compound 5 and $\mathbf{6}$ were isolated from ethyl acetate fraction. The ${ }^{13} \mathrm{C}-\mathrm{NMR}$ and DEPT spectra of compounds 5 and 6 showed signals of a carbonyl group $\left(\delta_{\mathrm{C}} 169.1-170.5, \mathrm{C}-7\right)$, two pairs of equivalent carbons at $\delta_{\mathrm{C}} 110.4$ (d, C-2, C-6), 146.5 (s, C-3, C-5) and two quarternary carbons at $\delta_{\mathrm{C}} 139.7$ (s, C-4) / 122.0 (s, C-1), belonging to a 3,4,5-trisubsituted benzoic acid derivative. The ${ }^{1} \mathrm{H}$ - and ${ }^{13} \mathrm{C}-\mathrm{NMR}$ spectra also showed the presence of a methyl group in compound 6. From spectroscopic evidences, compound 5 was identified as 3,4,5-trihydroxy benzoic acid or gallic acid [13] and compound $\mathbf{6}$ was its derivative as 3,4,5-trihydroxymethylbenzoate or methyl gallate [14].

Compound 7 was also obtained from $n$-hexane fraction. The ${ }^{1} \mathrm{H}$ - and ${ }^{13} \mathrm{C}$-NMR spectra of 7 were similar to those of gallic acid 5, except for the presence of a proton substituted for an hydroxy group at carbon $\mathrm{C}-5\left(\delta_{\mathrm{C}} 117.8\right)$. In agreement with that, the ${ }^{1} \mathrm{H}-\mathrm{NMR}$ spectrum of 7 showed signals of three aromatic methine protons at $\delta_{\mathrm{H}} 7.45(\mathrm{~s}, \mathrm{H}-2), 6.81(\mathrm{~d}, J=8.0 \mathrm{~Hz}, \mathrm{H}-5)$ and 7.44 (d, $J=8.0 \mathrm{~Hz}, \mathrm{H}-6$ ), belonging to a 3,4-disubsituted benzoic acid. In comparison to literature, compound 7 was identified as 3,4-dihydroxybenzoic acid, trivial name protocatechuic acid.

Compound 8, obtained as white powder from $n$-hexane fraction, was elucidated as $\beta$ sitosterol on the basis of the NMR data and comparison with the data reported in the literature [15].

Compound 9 was obtained as colourless oil from $n$-hexane fraction of methanol extract of C. citrinus leaves. From ESI-MS ion peak at $\mathrm{m} / z 205[\mathrm{M}+\mathrm{H}]^{+}$, the molecular fomular of 8 was identified as $\mathrm{C}_{15} \mathrm{H}_{24}(\mathrm{M}=204)$. The ${ }^{1} \mathrm{H}$ NMR spectrum of 9 contained the signals of four quartenary methyls at $\delta_{\mathrm{H}} 1.68\left(3 \mathrm{H}, \mathrm{s}, \mathrm{H}_{3}-15\right) / 1.60\left(9 \mathrm{H}, \mathrm{s}, \mathrm{H}_{3}-12, \mathrm{H}_{3}-13\right.$ and $\left.\mathrm{H}_{3}-14\right)$, five methylene at $\delta_{\mathrm{H}} 1.99-2.07$; and two methines at $\delta_{\mathrm{H}} 5.10(2 \mathrm{H}, \mathrm{s}, \mathrm{H}-2,-10)$. These data, together with the presence of 15 carbon signals in the ${ }^{13} \mathrm{C}$ NMR spectrum $\left(4 \times \mathrm{C}_{4}, 2 \times \mathrm{CH}, 5 \mathrm{x} \mathrm{CH}_{2}, 4 \mathrm{x}\right.$ $\mathrm{CH}_{3}$ ) suggested that 9 was a sesquiterpene. By comparison of the spectroscopic data with those published in literature, compound 9 was identified as 2,6,10-bisabolatriene [16].

\section{CONCLUSION}

In the search for bioactive constituents from Vietnamese plants, the leaves and stems of Callistemon citrinus (Curtis) Skeels were selected for chemical investigation. From the methanolic extract of this species, solvent-soluble fractions with increased polarity were produced including $n$-hexane, dichloromethane, ethyl acetate and water. Phytochemical analysis of different fractions of the plant led to the isolation of eight phenolic compounds including two flavones (eucalyptin (1) and 8-demethyleucalyptin (2)), two alcohols (blumenol A (3), ntetratriacontanol (4)), three benzoic acid derivatives (acid gallic (5), methyl gallate (6) and protocatechuic acid (7)), one sterol $(\beta$-sitosterol (8)), along with one sesquiterpene $(2,6,10$ bisabolatriene (9)). The structures of the natural compounds were determined from the spectroscopic evidences including 1D- and 2D-NMR and ESI-MS.

Acknowledgement. This research was supported by Ministry of Science and Technology (MOST) for Vietnam-Korea Project (Code 52/2011/NĐT). 


\section{REFERENCES}

1. Pham Hoang Ho, ed. An Illustrated Flora of Vietnam Vol. II. 2000, Youth Publisher: Hanoi, Vietnam. p. 41.

2. Nazreen S., Kaur G., Alam M. M., Shafi S., Hamid H., Ali M. and Alam M. S. - New flavones with antidiabetic activity from Callistemon lanceolatus DC, Fitoterapia 83 (8) (2012) 1623-1627.

3. Khambay B. P. S., Beddie D. G., Hooper A. M., Simmonds M. S. J., and Green P. W. C. New Insecticidal Tetradecahydroxanthenediones from Callistemon viminalis, J. Nat. Prod. 62 (12) (1999) 1666 -1667.

4. Rattanaburi S., Mahabusarakam W., Phongpaichit S., and Carroll A. R. Acylphloroglucinols from Callistemon lanceolatus DC, Tetrahedron 69 (30) (2013) 60706075 .

5. Jeong W., Hong S. S., Kim N., Yang Y. T., Shin Y. S., Lee C., Hwang B. Y. and Lee D. Bioactive triterpenoids from Callistemon lanceolatus, Arch. Pharm. Res. 32 (6) (2009) 845-849.

6. Rattanaburi S., Mahabusarakam W., Phongpaichit S. and Carroll A. R. - Neolignans from Callistemon lanceolatus, Phytochemistry Lett. 5 (1) (2012) 18-21.

7. Goyal P. K., Jain R., Jain S. and Sharma A. - A Review on biological and phytochemical investigation of plant genus Callistimon, Asian Pac. J. Trop. Biomed. 2 (3, Supplement) (2012) S1906-S1909.

8. Khanh P.N., Duc H.V., Huong T.T., Son N.T., Ha V.T., Van D.T., Tai B.H., Kim J.E., Kim Y.H. and Cuong N.M. - Acylphloroglucinol derivatives and triterpenoids with soluble epoxide hydrolase inhibitory activity from Callistemon citrinus,Fitoterapia (submitted) (2015).

9. Khanh P.N., Duc H.V., Huong T.T., Son N.T., Ha V.T., Van D.T., Tai B.H., Kim Y.H. and Cuong N.M. - Flavonoids and triterpenoids with inhibitory effect on NO production in LPS-activated RAW264.7 macrophage from Callistemon citrinus, Bull. Korean Chem. Soc. (submitted) (2015).

10. Sidana J., Neeradi D., Choudhary, A., Singh S., Foley W. J. and Singh I. P. Antileishmanial polyphenols from Corymbia maculata, J. Chem. Scie. 125 (4) (2013) 765-775.

11. Wu D.-K., Zhang C.-P., Zhu C.-Y., Wang Y.-L., Guo L.-L., Zhang K.-Q. and Niu X.-M. Metabolites from Carnivorous Fungus Arthrobotrys entomopaga and Their Functional Roles in Fungal Predatory Ability, J. Agr. Food Chem. 61 (17) (2013) 4108-4113.

12. Shukla Y.N. and Thakur R.S. - Heptatriacontan-4-one, tetratriacontanyl octacosanoate and other constituents from Pedalium murex, Phytochemistry 22 (4) (1983) 973-974.

13. Liu Y., Pukala T.L., Musgrave I.F., Williams D.M., Dehle F.C. and Carver J.A. - Gallic acid is the major component of grape seed extract that inhibits amyloid fibril formation , Bioorg. Med. Chem. Lett. 23 (2013) 6336-6340.

14. Wang C.R., Zhou R., Ng T.B., Wong J.H., Qiao W.T. and Liu F. - First report on isolation of methyl gallate with antioxidant, anti-HIV-1 and HIV-1 enzyme inhibitory activities 
from a mushroom (Pholiota adiposa).,Environ. Toxicol. Pharmacol. 37 (2) (2014) 626637.

15. Carle R., Beyer J., Chemina A. and Krempp E. 2H NMR determination of site-specific natural isotope fractionation in (-)- $\alpha$-bisabolols, Phytochemistry 31 (1) (1992) 171-174.

16. Lee T-H., Chiou J-L., Lee C-K. and Kuo, Y-H. - Separation and determination of chemical constituents in roots of Rhus javanica L. var. roxburghiana, J. Chinese Chem. Soc. 52 (2005) 833-841.

\section{TÓM TẮT}

\section{CÁC HỢP CHẤT PHENOL PHÂN LẬP TỪ LÁ VÀ CÀNH LOÀI CALLISTEMON CITRINUS}

Phạm Ngọc Khanh ${ }^{1}$, Hồ Việt Đức ${ }^{2}$, Trần Thu Hường ${ }^{1}$, Vũ Thị Hà ${ }^{1}$, Đoàn Thị Vân ${ }^{1}$, Ninh Thế Sơn ${ }^{1}$, Young Ho Kim³ ${ }^{3}$, Nguyê̂n Mạnh Cường ${ }^{1, *}$

${ }^{1}$ Viện Hóa học các hợp chất thiên nhiên, Viện Hàn lâm Khoa học và Công nghệ Việt Nam, 18 Hoàng Quốc Việt, Cầu Giấy, Hà Nội

${ }^{2}$ Khoa Dược, Trường Đại học Y Dược Huế, Đại học Huế, 06 Ngô Quyền, Huế, Việt Nam

${ }^{3}$ Khoa Dược, Trường Đại học Quốc gia Chungnam, Daejeon 305-764, Hàn Quốc

"Email: nmcuong_inpc@yahoo.com.vn

Từ lá và cành loài Tràm bông đỏ Callistemon citrinus (Curtis) Skeels tám hợp chất phenol và một hợp chất sesquiterpene đã được phân lập và xác định cấu trúc bao gồm hai flavon (eucalyptine (1) và 8-demethyleucalyptine (2)), hai hợp chất alcol (blumenol $\mathrm{A}(3), n$ tetratriacontanol (4)), ba dẫn xuất của acid benzoic (acid gallic $(\mathbf{5})$, methyl gallate $(\mathbf{6})$ và acid protocatechuic (7)), một hợp chất sterol $(\beta$-sitosterol $(\mathbf{8}))$ và một sesquiterpene $(2,6,10$ bisabolatriene (9)). Cấu trúc hóa học của các hợp chất trên được xác định nhờ các phương pháp hóa lý và phương pháp phổ bao gồm phổ cộng hưởng từ nhân 1 chiều, 2 chiều và phổ khối lượng.

Tù khóa: Callistemon citrinus (Curtis) Skeels, flavonoit, triterpenoit. 